\title{
¿Existe la filosofía o sólo es un fantasma sin valor real? Un análisis de la importancia de la filosofía en el tiempo contemporáneo.
}

\author{
DOI: http://dx.doi.org/10.5377/koot.v0i9.5909
}

URI: http://hdl.handle.net/11298/439

Marcelino R. Rojas

Docente de Filosofía Escuela de Antropología Universidad Tecnológica de El Salvador rojas.marcelino@gmail.com

"El gran pensamiento filosófico es el resultado más sublime de una vida infinitamente escogida."

Georg Simmel

\section{Resumen}

El presente artículo versa sobre la importancia de la filosofía para el conocimiento y para la práctica de la vida humana en el tiempo contemporáneo. Se distingue entre dos aspectos o temas de estudio en la filosofía, su índole de conocimiento y su importancia. Para la índole de la filosofía se utiliza como ejemplo a Xavier Zubiri. Y para inferir la importancia de la filosofía el pensamiento de Georg Simmel. Para Zubiri la filosofía es rigurosamente metafísica o filosofía de la realidad. Y desde Simmel, se optó por enfatizar las ideas que nos señalan algunas pistas sobre la importancia de la filosofía. Sirva el presente artículo como un tema abierto a discusión y a disensión.

Palabras claves: Conocimiento mítico, conocimiento científico, conocimientos filosóficos, presupuestos del conocimiento, conocimiento verdadero, ética, moral.
Abstract
The current article deals with the importance of philosophy for knowledge and for the practice of human life in contemporary times. It distinguishes between two aspects or topics of study in philosophy: its nature of knowledge and its importance. Xavier Zubiri ist taken as the reference of the nature of philosophy. Then, the thinking of Georg Simmel in order to infer the importance of philosophy. On one hand, Zubiri philosophy is rigorously metaphysical or philosophy of reality. On the other hand, Simmel chose to emphasize the ideas that show us some clues about the importance of philosophy. This article has been designed as a topic open to discussion and dissension. 
Keywords: Mythical knowledge, scientific knowledge, philosophical knowledge, previous knowledge, true knowledge, ethics, moral.

\section{Introducción}

Hay distintos aspectos de la filosofía que pueden ser abordados como temas de estudio, por ejemplo la posibilidad misma de la filosofía, su índole de conocimiento, el valor o importancia de la filosofía, entre otros. En el presente artículo elegí tomar como ejemplo de estudio de la índole de la filosofía a Xavier Zubiri y como base para el estudio de la importancia de la filosofía al pensamiento de Georg Simmel. También, el aspecto de la posibilidad de la filosofía aparecerá, pero en función y en referencia al énfasis principal del presente escrito: la importancia de la filosofía en el tiempo contemporáneo.

La filosofía históricamente ha abordado una diversidad de zonas de la realidad, de objetos de estudio, de métodos y de problemas. Sin embargo, en el presente artículo no se trata de determinar cuáles son éstos ni cuáles son sus contenidos ni sus diversas estructuras (Zubiri, 1992). Y, también, existe una diversidad de filósofos en la historia de la filosofía que constituyeron con su misma actividad, más implícita que explícitamente, el valor de la filosofía: presocráticos, Sócrates, Platón, Aristóteles, Tomás de Aquino, Descartes, Kant, Hegel, Nietzsche, Marx, Bergson, Husserl, Heidegger, Simmel, Wittgenstein, Habermas, Apel, Zubiri, entre muchos otros. Y cada uno de estos filósofos tiene su propia idea de la posibilidad, de la índole y del valor de la filosofía. Lo mismo que cada uno determinó para sí qué es la filosofía, su objeto, su método y sus problemas.

Y lo que es todavía más radical, cada uno define de manera distinta los mismos conceptos. Distintos filósofos pueden tratar de lo mismo, pero no decir lo mismo (Zubiri, 1992). Pero, como el deseo del presente artículo no es el desarrollo de todos los temas o aspectos que se pueden tratar de y en la filosofía, y tampoco tratar a todos los autores, voy a limitarme a dos autores, Xavier Zubiri y Georg Simmel, que nos sugieren una serie de ideas sobre la índole y la importancia de la filosofía para el tiempo contemporáneo. Advierto que en el desarrollo de la importancia de la filosofía he tomado como referencia el libro de Simmel "Problemas fundamentales de la Filosofía".

En el presente escrito realizaré una exploración e inferencias sobre las ideas de esta obra, que descubre la importancia de la filosofía en el tiempo contemporáneo. Por tanto, el presente escrito no es una mera repetición de dicho autor, sino que parándome sobre los hombros de este gigante intento ver más allá de su mirada y realizo afirmaciones con las que quizá sí o quizá no estaría de acuerdo el mismo Simmel, pero que expreso en el presente artículo esperando que el lector analice y valore, sin esperar que necesariamente comparta o no mis ideas. Este autor es Georg Simmel. 


\section{¿Quién es Georg Simmel?}

Georg Simmel, de origen alemán, nació en Berlín en 1858 y murió en Estrasburgo, en 1918. Escritor de 31 libros y más de doscientos artículos. Amigo de Husserl. Fundador con Max Weber de la Sociedad Sociológica Alemana en 1910.

\section{Un primer acercamiento a la filosofía}

¿Multiplicidad o unidad de la filosofía?

Es un hecho histórico la existencia de una multiplicidad de definiciones abarcadas por el concepto de filosofía. Parafraseando se puede afirmar que para gustos hay muchas filosofías y para filosofías hay muchos gustos. Sin embargo, es sumamente ingenuo que en esta diversidad de definiciones de la filosofía no se encuentre una estructura que vincule a las distintas concepciones de filosofía a alguna unidad. La determinación de una estructura básica de la multiplicidad de ideas abarcadas por el concepto de filosofía es un problema y una tarea a resolver por la misma filosofía. Un esfuerzo contemporáneo por responder a este problema, digno de mencionar como caso y ejemplo, proviene de Xavier Zubiri.

\section{La filosofía en Zubiri como rigurosa metafísica}

Zubiri aborda este problema de la multiplicidad o unidad de la filosofía distinguiendo en la idea de filosofía, sus estructuras de sus contenidos. De acuerdo a Zubiri es posible descubrir las estructuras de la filosofía y esta es una tarea de la misma filosofía (Zubiri, 1992). Pero, a pesar de identificar las distintas estructuras de la filosofía, el problema de la filosofía sigue estando latente: qué pueden tener en común todas las diversas filosofías para llamarse filosofía. Y esta pregunta sólo se puede esclarecer mediante el esfuerzo filosófico de un filósofo que se plantea el problema, aunque no conozca la respuesta. Si se quiere respuestas, se tienen que buscar. Y la vía que seguirá Zubiri para buscar una respuesta es el camino mismo recorrido históricamente por los mismos filósofos.

Quiero hacer notar que el mismo Zubiri afirma de entrada que la filosofía es constitutivamente un saber en marcha (Zubiri, 1992). La filosofía tiene unas estructuras aunque no los mismos contenidos. La filosofía no está hecha de una sola vez y para siempre, sino que va siendo construida históricamente, sobre el examinar distintos objetos con distintos métodos. ¿Es que acaso, la física u otra de las llamadas ciencias se han construido y se construyen de otra manera? Tal parece que las diferencias en la filosofía muchas veces vienen determinada por las elecciones de un determinado autor sobre un determinado objeto y sobre un determinado método de examinar dicho objeto.

La misma filosofía tiene distintos horizontes de intelección y dentro de un mismo horizonte la filosofía ha adoptado diversas estructuras. Por ejemplo, para 
Zubiri (1992), en el caso de la filosofía europea, ésta tiene dos horizontes de intelección: el de la filosofía griega (el horizonte del movimiento, el cambio) y el horizonte de la nihilidad (hay cosas en lugar de nada). Y dentro de un mismo horizonte la filosofía ha adoptado diversas estructuras: La filosofía como una forma de vida (cínicos, cirenaicos y estoicos), la filosofía como una doctrina de la vida (cuál es el sentido de la vida), la filosofía como un conocimiento de las cosas (incluidos el hombre y su vida) (Zubiri, 1992). Sin embargo, sigue estando en suspenso una respuesta acerca de qué sea la filosofía más allá de la diversidad de las llamadas filosofías.

Frente a la diversidad de saberes, ya sean empíricos, técnicos, morales, y especialmente científicos, Zubiri, siguiendo su disciplina de rigurosidad histórica del desarrollo del conocimiento filosófico, afirma que la índole de la filosofía es rigurosamente metafísica (Zubiri, 2004). Para Zubiri, la metafísica es un sistema abierto. Esto significa, que no es una realidad acabada ni un sistema de conceptos, sino un sistema de estructuras que hay que ir aprendiendo estructuralmente -con mucho esfuerzo-, las unas después de las otras, y con todas ellas construir -y enriquecer- problemáticamente la idea de realidad en cuanto tal (Zubiri, 2001). Para Zubiri, metafísica es, al hilo de la impresión de la realidad, filosofía de la realidad (Zubiri, 1980). Es lo físico mismo, pero en dimensión formal distinta. $\mathrm{O}$ dicho con mayor rigor y precisión, es lo físico pero en función trascendental. El mismo Zubiri insiste que se trata de una concepción unitaria de lo real en impresión de realidad. Esto significa que el momento de realidad y su trascendentalidad son estricta y formalmente físicos.

Lo metafísico no es un allende lo físico, sino lo físico mismo: es aprehensión sintiente de la física trascendentalidad de lo real (Zubiri, 1980). Sin embargo, lo metafísico pende de lo físico, por ejemplo, la metafísica es abierta porque la realidad es abierta y no al revés. Y en tanto la realidad es dinámicamente abierta, la metafísica también. Zubiri entiende la metafísica como saber trascendental de, en y por las cosas mismas. La filosofía puede tratar sobre las mismas cosas reales que tratan los técnicos y los científicos, pero desde su trascendentalidad: más allá de sus contenidos, esto es desde sus estructuras. La respuesta de Zubiri es todo lo discutible que nos podamos imaginar. Pero sirva esta como un ejemplo de respuesta de la índole de la filosofía.

\section{La idea de filosofía en Simmel como un torrente viviente y creador}

Sin embargo, la filosofía puede ser comprendida, también, como un torrente viviente y creador (Simmel, 2006). Infiriendo, la filosofía puede ser comprendida como un proceso creativo de carácter histórico, y no como una respuesta a encontrar. Personalmente, y empleando una metáfora, pienso que la filosofía tiene la virtud de abrir la mirada y de iluminar aquellos oscuros rincones que se encontraban ocultos, incluida la ampliación de su propia mirada y la iluminación de sus propios rincones oscuros. Utilizando otra comparación, en el tiempo contemporáneo la filosofía misma ha de rescatarse de la garra de los 
productos (imágenes y representaciones, entre otras consideradas definitivas) para mantenerse dentro del torrente vivo del espíritu humano (en un proceso espiritual creativo).

Para Simmel, existe un complejo de pensamientos abarcados por el concepto filosofía (Simmel, 2006). Y la posibilidad de determinar qué sea la filosofía sólo lo puede realizar ella misma creando sus propios instrumentos y medios. Sin embargo, este mismo autor es todavía más radical, y a esto quiero que presten atención: Simmel llega hasta plantearse el problema de si ¿existe algo a lo que llamamos filosofía o la filosofía sólo cubre con su nombre un fantasma sin valor real? (Simmel, 2006). Y por supuesto se puede negar a la filosofía su propia realidad o su estatuto o índole dentro de lo que llamamos conocimientos con un valor real. Este problema sobre la índole de la filosofía suele definirse como ¿qué tipo de conocimiento es la filosofía? Y por supuesto resulta bastante ligero descalificar de entrada a la filosofía y negarle cualquier valor real por la diversidad de objetos, métodos, finalidades y productos contenidos en el mismo concepto de filosofía.

Y no son pocos los que consideran que la misma tarea de preguntarse por aquello que es la filosofía es una pérdida de tiempo, porque la filosofía, justa y precisamente, no sirve para nada, o lo que es todavía más radical que no tiene ningún valor real dentro del mundo del conocimiento actual. Sin embargo, muchas veces no solemos caer en la cuenta de este valor de la filosofía, surgido de lo anteriormente dicho: "La filosofia tiene la capacidad de preguntarse hasta por su propia existencia como algo con valor real”. A los que niegan el valor de la filosofía, un auténtico filósofo, les da el beneficio de la duda, precisamente porque es un auténtico filósofo, cosa que no son sus detractores. Y esta capacidad de dudar y de sospechar muestra uno de los valores de la filosofía, al que no escapa ella misma como parte de ese torrente vivo y creativo del espíritu humano.

\section{La importancia histórica de la filosofía frente al conocimiento mítico, científico y frente a los mismos productos filosóficos}

Una diferencia fundamental histórica entre los conocimientos míticos y la filosofía, creados y desarrollados en Grecia, es que ésta última, la filosofía, se preguntó por las causas reales de los fenómenos de la naturaleza más allá de las explicaciones míticas que fundamentaban dichos fenómenos en la realidad $\mathrm{y}$ en la voluntad de los dioses. Recordemos que en el mundo del conocimiento mítico griego la caída de un rayo se debía a que había un Dios, Zeus, que lanzaba desde los cielos dicho rayo. Y lo normal es que este conocimiento se transmitiera de generación en generación hasta que un hombre, al que se le llamaría filósofo, se preguntó: ¿no será que hay otra causa a la caída de un rayo distinta a los relatos míticos? Este hecho constituyó el principio del proceso de desarrollo histórico de la filosofía y posteriormente de las ciencias. Se trata del movimiento histórico del conocimiento mítico al filosófico, como antecedente 
y condición del conocimiento científico. ¿Cuántos logran percibir el importante papel que jugaron los filósofos en este proceso histórico de descubrimientos y de construcción de lo que luego históricamente fue nombrado y es conocido actualmente como ciencias?

Siguiendo con la importancia de la filosofía frente al conocimiento científico, en la actualidad, por lo general, los conocimientos hoy llamados científicos se aprenden de la misma manera que los conocimientos míticos del mundo griego: como verdaderos conocimientos sin saber si realmente lo son. Para muestra de la falibilidad humana, incluida las ciencias, en la historia del saber de la humanidad durante años, décadas, siglos y hasta miles de años se aprendieron muchos conocimientos como verdaderos hasta que después se descubrió lo contrario. Cito como ejemplo la siguiente afirmación creída por filósofos y por científicos, y por más de mil años como verdadera: "la tierra es el centro del universo y alrededor de ella giran todos los astros".

Continuando con los productos filosóficos, y todavía siendo más radicales, suele ocurrir con los conocimientos llamados filosóficos lo mismo dicho para los mitos y para la ciencia: se aprenden y se enseñan como verdades sin saber si realmente lo son. Sin embargo, la misma filosofía, tiene esa virtud de cuestionarse a sí misma. Según Simmel (2006), esto es un proceder particular de la filosofía. Así que esta es una característica de la filosofía: su capacidad histórica para autocriticarse y autocorregirse a sí misma. ¿Apostaría usted por una filosofía que se autocrítica y autocorrige? ¿Una filosofía que juzga que ella misma podría estar equivocada, y por ello investiga y busca las pruebas de sus afirmaciones fundamentales y que las corrige si las encuentra equivocadas?

\section{La importancia de la filosofía como conciencia y autoconsciencia de los presupuestos del conocimiento y de un ir más allá de estos presupuestos}

Una pregunta que ayudará además a plantear mejor el valor de la filosofía es si la filosofía parte de la ausencia de una hipótesis o de los tan llamados presupuestos. En honor a la misma filosofía, por supuesto, que ninguna filosofía parte de la ausencia de hipótesis o presupuestos. La ausencia absoluta de hipótesis es inalcanzable. El que busca, parte de la impresión inmediata del mundo sensorial o de los valores morales tradicionales, de la evidente validez de la experiencia o hasta, inconscientemente, de los poderes divinos (Simmel, 2006). Así que, la filosofía no parte de cero. No es dado al hombre comenzar desde cero. Cuando la filosofía inicia su tarea encuentra dentro de sí o fuera de sí una realidad o un pasado, que ofrecen a su proceder una materia, un punto de partida, o por lo menos algo opuesto y que debe ser destruido (Simmel, 2006). Sin embargo, la filosofía es un ir más allá de "lo dado", de "lo encontrado". Esta es una constante aspiración de la filosofía. ¿Apostaría usted por una filosofía que no sólo es consciente de sus hipótesis y presupuestos, de sus limitaciones históricas, sino que va más allá de ellas? 


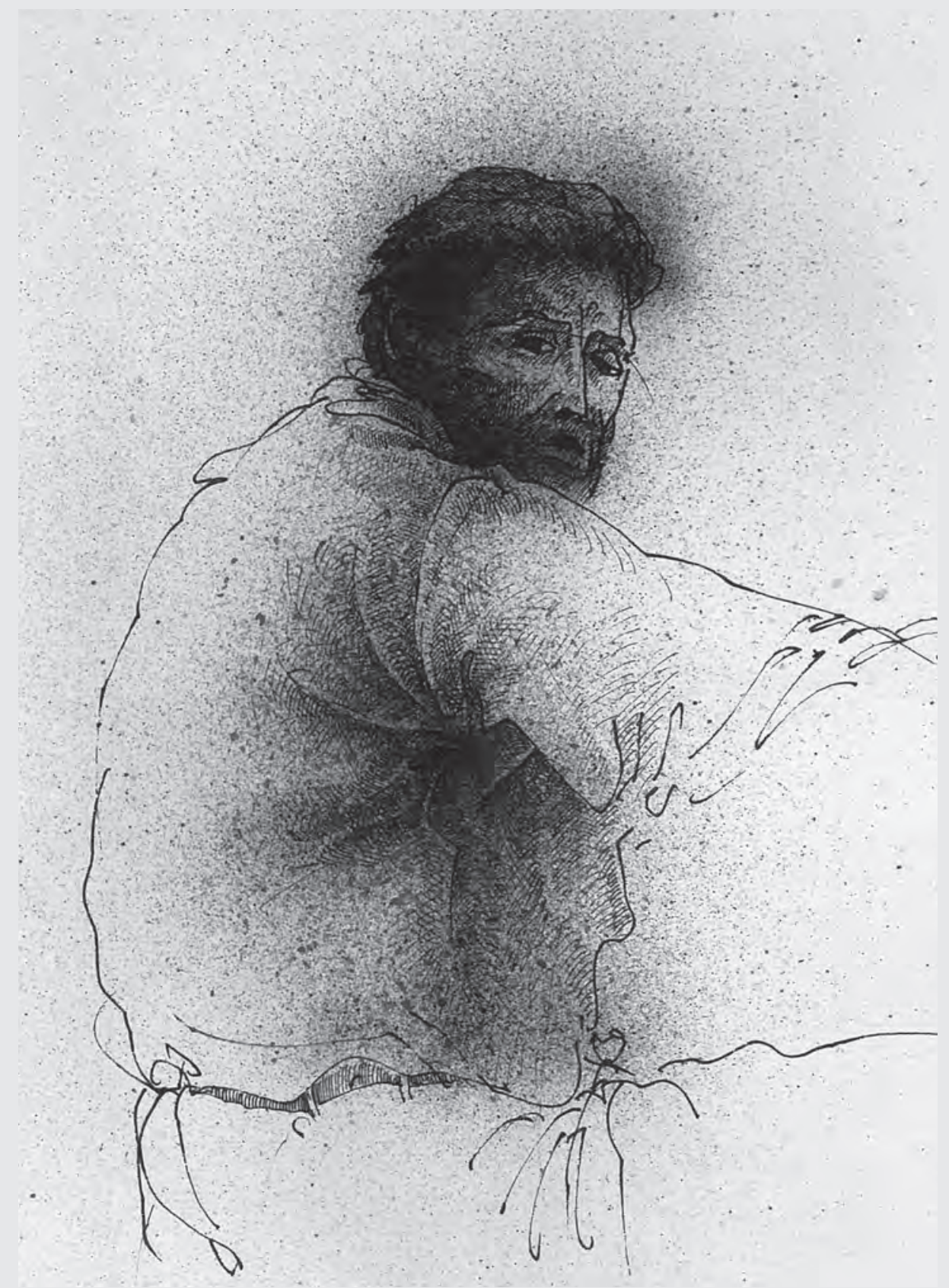

Augusto Crespín 


\section{La importancia de la filosofía frente al conocimiento verdadero}

Otra pregunta importante es si ¿La filosofía es capaz de alcanzar conocimientos que pueda tomar como verdaderos o reales? Para muestra, si se viene advirtiendo, una conclusión filosófica resultante de lo hasta ahora dicho, y citando a Simmel, es que "el hombre y su conocimiento están condicionados" por "algún dado o encontrado", o sea, por alguna premisa surgida de una experiencia sensorial o recibida de una tradición cultural, religiosa o científica. De esta premisa, o más bien premisas, ya sean o no realidades externas, ya sean o no leyes interiores, depende en múltiple limitación la soberanía, el contenido y la tendencia del conocimiento- sea ello tan sólo las reglas de la lógica y del método, o el hecho de un mundo existente- (Simmel, 2006).

La importancia de la filosofía frente a los "presupuestos" que condicionan y determinan el conocimiento y hasta la práctica de la vida humana misma. Y, todavía, yendo más allá de Simmel, se podría ser más radical afirmando que "cualquier conocimiento humano está determinado por algún dado o encontrado". Y que por supuesto determina no sólo el desarrollo del mismo conocimiento, sino también la práctica de la vida humana misma. Sin embargo, se debe aclarar que esta determinación no significa que exista un único "algún dado o encontrado".

Al contrario, la filosofía no sólo descubre este "algún dado o encontrado", sino además, la existencia de una diversidad de "algún dado o encontrado", pero que ya sea uno u otro, cualquier dado o encontrado afecta al conocimiento y hasta las diversas actividades de la vida humana misma. He aquí otro de los valores reales de la filosofía: descubrir cuáles son estos "dados o encontrados". Esta es otra función de la filosofía que pasa desapercibida y que es necesario realzar como un hecho a favor del valor de la filosofía. ¿Apostaría usted por una filosofía que nos descubre cuáles son estos "dados y encontrados" que condicionan y determinan el conocimiento y hasta la práctica de la vida humana misma?

La importancia de la filosofía como des-ocultamiento: sacar a la luz lo oculto.

Avanzando con Simmel, donde quiera que se inicie el conocimiento existe ya algo supuesto, que nos inquieta como tinieblas insuperables o, por el contrario, es un punto de apoyo en la relatividad del mismo conocimiento (Simmel, 2006). Y como se indicó arriba, a la filosofía le corresponde descubrir cuáles son esos supuestos -hipótesis- que guían la actividad científica e incluso la actividad misma de la filosofía. Valiosa e inigualable virtud de la filosofía: des-velar o desocultar (sacar a la luz lo oculto, ya sea ocultado consciente o inconscientemente). Continuando con la importancia de la filosofía, recordemos y tomemos como un hecho que a la hora de definir a la filosofía encontramos una diversidad histórica de respuestas, tantas como autores han ido apareciendo en la misma 
historia humana. Y, por supuesto, no sólo esto, sino también, en la misma historia de la filosofía encontramos una diversidad de preguntas, de métodos y de respuestas, y de la misma manera tantos objetos de estudio, tantas preguntas, tantos métodos y tantas respuestas como filósofos se encuentran en la misma historia de la filosofía. A simple vista, se puede interpretar este hecho como una debilidad de la filosofía y, en el peor de los casos, como una razón para descartar a la filosofía, como ya se intentó en el pasado cercano histórico, y como se sigue intentando en el presente. No necesitamos de la filosofía porque ya poseemos ciencias. Ciencias que tienen unas preguntas, métodos y respuestas definidas. Y, por ende, se afirma alegremente: "ya no hace falta la filosofia". Sin embargo, nada más contrario al espíritu de libertad del hombre, libertad para pensar distinto, libertad de crear algo nuevo, libertad de expresar lo diferente, libertad de ser único, diverso.

Y en la existencia de la filosofía está en juego esta libertad de la vida humana misma: libertad de pensar, libertad de expresar y libertad de actuar. Y es la filosofía la que sigue luchando contra los nuevos mitos del presente que se aprehenden, se toman como verdaderos y se transmiten históricamente de una generación a otra sin cuestionar su verdad y su bondad. Por ejemplo, uno de estos nuevos mitos tiene que ver con el cientificismo, que da por hecho que un conocimiento por provenir de las actividades de los llamados, y autollamados científicos, es de por sí y en sí verdadero. Nada mejor que la filosofía para hacer una crítica a esta falsa pretensión de la ciencia, que en contra de los hechos no es capaz de ver y reconocer las equivocaciones históricas de muchos científicos, incluida la autollamada comunidad científica, quienes pasaron por verdadero unos supuestos, que con el paso del tiempo, se descubrieron conocimientos falsos. ¿Apostaría usted por una filosofía que ha ayudado a las ciencias a caer en la cuenta de sus equivocaciones o desviaciones?

\section{La importancia de la filosofía como teoría del conocimiento y como un campo vital de autonomía y libertad}

Retomando el tema, una de las desembocaduras de la filosofía es la teoría del conocimiento, en la que las ciencias actuales y la misma filosofía son puestos como objeto de estudio. La filosofía se encarga de "sacar a luz" los supuestos -hipótesis-, que dichas ciencias y la misma filosofía dan por hecho como verdaderos. ¿A quién le corresponde esta tarea? Por supuesto, a la filosofía. Sin embargo, históricamente, la filosofía se ha dado a sí misma no sólo esta tarea u objeto de estudio, sino una multiplicidad de tareas u objetos de estudio, lo mismo que métodos. Hay que decir, que la filosofía sigue siendo un campo de libertad humana, un campo de libertad para equivocarse, un campo de libertad para aprender de nuestros errores. Quiénes están dispuestos a defender este campo de libertad no sólo defienden a la filosofía, sino a las mismas ciencias, que históricamente han avanzado por tanteo y error, y a la propia libertad de la vida en sociedad, una sociedad abierta. En este punto, es necesario ser capaces de ver la importancia de la filosofía no sólo para las ciencias, sino también para 
la vida individual y para la vida en sociedad de esos mismos individuos. Esta falta del campo vital de la libertad humana de la filosofía tiene consecuencias gravísimas que evidencian el enriquecimiento o el empobrecimiento de la vida de las personas y de los pueblos. Hemos de recordar la altura humana y social que alcanzaron los griegos gracias a la filosofía. Tanto así que la altura de un pueblo se ha medido históricamente por la producción de conocimientos de sus filósofos. ¿Apostaría usted por este campo vital de la libertad humana al que se le llama filosofía?

Para Simmel, la autonomía interior de la filosofía trae como consecuencia que ella determine su problema, su objeto, sus finalidades y métodos (Simmel, 2006). Este hecho determina la diversidad de objetos de estudio, de problemas, de métodos y de respuestas. Este es un derecho y un deber de la filosofía misma. De aquí que cada pensador -filósofo- pueda ser original en las preguntas, en los objetos, en los métodos y en las respuestas. En este punto, pregunto a mis lectores: ¿Alguien quiere defender esta libertad creativa de la filosofía? ¿Acaso es poco el valor que se pone en riesgo al abandonar la actividad filosófica?

\section{La importancia histórica de la filosofía en la vida humana práctica y teorética}

Citando a Simmel, Epicuro define a la filosofía como un anhelo de alcanzar una vida dichosa a través de razones y meditaciones (Simmel, 2006). Por hoy quiero hacer hincapié en la filosofía como necesaria para una vida dichosa. ¿Apostaría usted por una filosofía que nos orienta hacia una vida dichosa?

Y Schopenhauer define a la filosofía como un anhelo de llegar por medio de imaginaciones a lo que no es imaginación, es decir, a lo trascendental del fenómeno empírico, del que se ocupan las restantes ciencias (Simmel, 2006). En este punto, también quiero recordar el papel de la necesaria imaginación puesta en la tarea histórica de los descubrimientos científicos. Además, históricamente a las llamadas ciencias no les ha hecho falta como base alguna filosofía y hasta también alguna teología, ya sean llamadas, por unos y por otros, conservadoras o progresistas. ¿Apostaría usted por una filosofía que le descubre a las ciencias los supuestos en que se funda y que no están a su vista?

Continuando con un ejemplo histórico, en la edad media la filosofía fue definida como sierva de la teología, y añado que lo mismo ocurrió en la edad moderna -y esto es una hipótesis-: la práctica de la filosofía fue definida por algunos sectores de pensadores como sierva de la ciencia; y, en la edad contemporánea, la filosofía es definida como sierva de la política. Y tal parece -y esto continúa siendo parte de la misma hipótesis- que en la actualidad algunos sectores de pensadores, traicionando a la filosofía, consciente e inconscientemente, siguen empleando a la filosofía para fundamentar y defender sus "verdades religiosas, científicas y políticas". Esto requiere de poner manos a la obra a otra función de la filosofía: la de sospechar, descubrir, "sacar a la luz", los supuestos que 


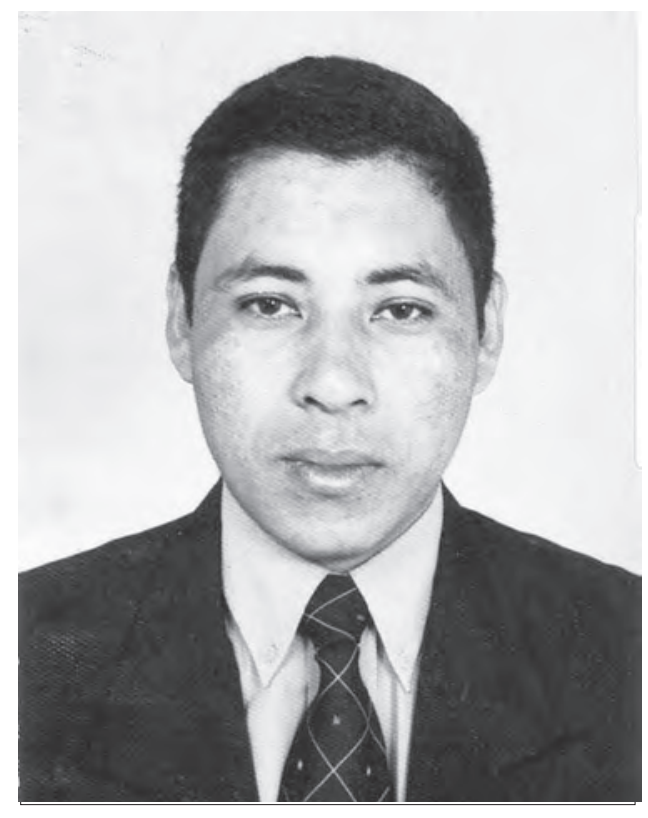

Máster y Filósofo Marcelino R. Rojas

fundamentan las llamadas verdades "religiosas", "científicas" y "políticas", y que no están a la vista. ¿Apostaría usted por una filosofía así?

Para Simmel, la misma filosofía se debe enfrentar con un nuevo problema: si cada filosofía singular es un producto de un pensador singular, ¿Qué tienen en común estos productos para que posean el mismo nombre de filosofía? (Simmel, 2006) En las preguntas, en los objetos de estudio, en los métodos y en las respuestas puede que no tengan nada en común. Pero, el filósofo, a diferencia de otros, en diversos grados, y nunca con perfección, realiza la misma búsqueda por traducir en conceptos y definiciones una comprensión racional -un sentido-, una intuición de la totalidad de las cosas y la vida misma (Simmel, 2006). Lo que está en juego en la filosofía es la misma búsqueda racional por las causas reales de la diversidad de problemas y la búsqueda de posibles y diversas soluciones a estos mismos problemas. Tal vez haya algunos o tal vez haya muchos que desprecien a la filosofía lo mismo que a la racionalidad humana. Sin embargo, en la actualidad, ¿alguno acusaría acertadamente a los malos espíritus de ser la causa del dengue o de la chikungunya?

La importancia de la filosofía como un esfuerzo histórico por traducir en conceptos y definiciones una comprensión racional determinada del mundo o de la vida

He aquí algo que tiene en común cualquier filosofía singular con otras filosofías singulares: este esfuerzo por traducir en conceptos y definiciones una comprensión racional determinada del mundo o de la vida. A cada 
cosa y a cada hombre en el mundo se le puede formular una pregunta y a cada pregunta se le formulan unas respuestas. $\mathrm{Y}$ todas estas respuestas son históricas, con todas sus casualidades, todos sus ajustes, todos sus extravíos, todos sus retrocesos, todas sus irregularidades y todas sus deficiencias. A este tipo de respuestas también corresponden las filosóficas, muchas veces, unilateralmente individuales, singulares y deficientes, encadenadas a factores históricos de su época. Sin embargo, con las ciencias tampoco ocurre de otra manera. Y es la filosofía misma la que nos hace caer en la cuenta de la historicidad y limitación de nuestras mismas preguntas y respuestas, incluidas las que se les llama científicas.

Un ejemplo claro de esto es la historia misma de aquello que durante una época se ha llamado científico y que luego de años, décadas, siglos y milenios se descubre como falso o errado. Así es que, el humano sólo posee en cada época histórica un determinado conocimiento, unos modelos inconclusos, en contante modificación y nunca susceptibles de ser concluidos para un ser histórico como el humano. De este tipo de índole de conocimiento son las ciencias y la filosofía. Esta última idea es un logro de la filosofía como intuición de la totalidad y no de las ciencias en su particularidad.

Según Simmel, en medio de esas infinitas relaciones de multiplicidad de las individualidades y de los destinos, de las experiencias y de las disposiciones anímicas de los humanos, se ha logrado un mínimo de verdaderos conceptos totales y pensamientos de unidad frente a la existencia (Simmel, 2006). Esto es lo que posibilita y permite la existencia de lo que llamamos ciencias, religiones y filosofías históricas. Y es la filosofía la que nos permite llegar a este conocimiento, parcial y fragmentario, sobre la realidad histórica, tanto de las ciencias como de las diversas filosofías.

\section{La importancia histórica de la filosofía como vínculo de unidad frente a la inmensurable multiplicidad, variedad, desmembración e irreconciliabilidad del mundo}

Para Simmel, el mundo nos es dado como un conjunto de fragmentos y la aspiración de la filosofía consiste en presentar el todo por la parte, lo que obtiene colocando la parte por el todo (Simmel, 2006). Históricamente, la filosofía ha ofrecido la unidad necesaria al humano frente a la inmensurable multiplicidad, variedad, desmembración e irreconciliabilidad del mundo; ha ofrecido una unidad en todas las confusiones y oposiciones del mundo de los fenómenos, un punto donde la extrañeza de las realidades cede ante su afinidad. Y nuestra época histórica no es la excepción. Sin embargo, esta imagen general del mundo es el producto de la relación entre una individualidad y sus circunstancias históricas. La visión de la totalidad sólo es posible para Dios. Esto no implica que el humano, llamado científico o filósofo, no vaya descubriendo nuevos fragmentos, aclaraciones, reconciliaciones en el mundo de los fenómenos. Afirmar que no es dado a la filosofía el conocimiento total de la realidad es verdadero, pero dejar de 
continuar formulando preguntas filosóficas - problemas- y buscando respuestas filosóficas a esas preguntas -soluciones- es suicidio. ¿Se ha de apostar o se ha de renunciar a la filosofía por su falibilidad? ¿Es la equivocación o error causa suficiente para no continuar con la búsqueda de conocimientos verdaderos?

\section{La importancia histórica de la filosofía como desencantadora de hechizos conceptuales}

A través de toda la historia de la filosofía se desarrollaron, confundidos y entre mezclados, los conceptos de la universalidad, de la unidad, de lo fundamental de la realidad de las cosas. En ello reside el fatal hechizo experimentado siempre por el pensamiento a través de los conceptos universales (Simmel, 2006). Sirva de ejemplo de este hechizo tanto la misma filosofía como las distintas ciencias y las religiones. Todas ellas tienen un origen histórico y son reproducidas históricamente a la discreción de una o varias generaciones humanas, por mucho que se le quiera cargar con un absoluto del que carecen en realidad.

A la misma historia humana se le carga con una finalidad. Sin embargo, esta finalidad, universalidad, unidad o fundamentalidad de la historia humana es determinada por la decisión y voluntad de quienes detentan el poder, ya sea el poder económico sobre los medios de producción que determinan las condiciones materiales de vida propias y de los demás; o, ya sea el poder político sobre las instituciones del estado, o ya sea el poder cultural, entre otros. ¿Apostaría usted por esta función crítica de la filosofía frente a la adjudicación arbitraria de quienes detentan la autoridad en una sociedad determinada, ya sea el poder cultural, político o económico?

\section{La importancia histórica de la filosofía como respuesta a la necesidad humana ética de la unificación del mundo moral.}

Quiero utilizar e insistir en un ejemplo de tipo político. Este es el caso de las ideologías políticas partidarias, que siendo meros instrumentos para la toma del poder o para mantenerse en el poder, son promovidas como verdades y empleadas como dogmas, aunque se encuentren muy alejadas de la verdad, del bien común y de la justicia social. Lastimera realidad social la de muchos latinoamericanos, que caen ingenuos ante partidos políticos, que emplean los conocimientos, ya sean verdaderos o falsos, como instrumentos ideológicos para alcanzar el poder político en un país, y una vez en el poder, se dedican a vivir como reyes, en una vida holgada de lujos y despilfarro, a costa del uso irracional del dinero del erario público, de la sobre-explotación de millones de trabajadores que son mal pagados y de la destrucción de los recursos naturales.

Sin embargo, la filosofía advierte sobre la necesidad humana ética de la unificación del mundo moral, incluido y, sobretodo, el mundo moral político. Esta unificación del mundo moral es una tarea o función de la filosofía. Esta unificación del mundo moral pasa a convertirse en un fin inalcanzable, pero que 
sirve de horizonte para ir avanzando en una dirección de racionalidad en el uso de los recursos naturales, en el uso de los bienes del estado y en las relaciones en el mundo del trabajo, tanto en la producción como en la distribución de la riqueza: imagen y anhelo de un mundo ético.

\section{Conclusiones}

En el estudio de la filosofía misma se pueden distinguir dos aspectos: la posibilidad e índole de la filosofía y el valor o importancia de la filosofía.

La filosofía ha jugado un papel histórico frente al quehacer creador del conocimiento mítico, del conocimiento científico y de la misma producción de conocimiento filosófico.

La filosofía además de un producto histórico, puede ser comprendida como un torrente vivo y creador en continuo que hacer frente a la vida humana misma.

La importancia de la filosofía está determinada por la medida de su capacidad:

- Como crítica y autocrítica, correctiva y auto correctiva de la inconmensurable producción histórica de conocimientos llamados míticos, científicos y de los mismos conocimientos llamados filosóficos. La misma filosofía es falible y abierta.

- Como conciencia y autoconsciencia de los presupuestos del conocimiento, sean cuales sean, y de su ir más allá de estos presupuestos.

- Como un campo vital de autonomía y libertad en la vida humana práctica y teorética.

- Como un esfuerzo histórico por traducir en conceptos y definiciones una comprensión racional determinada del mundo o de la vida.

- Como vínculo de unidad frente a la inmensurable multiplicidad, variedad, desmembración e irreconciliabilidad del mundo.

- Como desencantadora de hechizos conceptuales.

- Como respuesta a la necesidad humana ética de la unificación del mundo moral.

- Para discernir los criterios que sirven para distinguir el conocimiento verdadero del no verdadero. 
- Para identificar y valorar los "presupuestos" que condicionan y determinan el conocimiento y hasta la práctica de la vida humana misma, y descubrirlos: sacar a la luz lo oculto.

- Y por último, sirva este artículo como ejemplo y pistas a desarrollar sobre la importancia de la filosofía, esfuerzo al que se pueden sumar otros autores y otros aportes.

\section{Referentes bibliográficos}

Simmel, G. Problemas Fundamentales de la Filosofia. Andulucía: Ed. Espuela de Plata. 2006.

Zubiri, X. Inteligencia Sentiente. Inteligencia y Realidad. Madrid: Alianza. 1980.

Zubiri, X. Cinco lecciones de filosofía. Madrid: Alianza. 1992.

Zubiri, X. Sobre la realidad. Madrid: Alianza. 2001.

Zubiri, X. Naturaleza, Historia, Dios. Madrid: Alianza. 2004. 